\title{
Quiste folicular inflamatorio en posición atípica: Revisión de la literatura a propósito de un caso
}

\author{
Follicular inflammatory cyst in atypical location: Case report and literature review.
}

\author{
Emilio Moreno ${ }^{1, a}$, Jorge Lolas $M^{2, a}$, Alfonso Vergara ${ }^{3, a}$, Agustin Márquez ${ }^{4, b}$.
}

\section{RESUMEN}

Objetivos: Mostrar la importancia de la histopatología en el diagnóstico del quiste folicular inflamatorio para un correcto tratamiento y la relevancia de una nomenclatura precisa.

Material y Métodos: Búsqueda en Pubmed, ScienceDirect, EBSCO-host de «odontogenic-cysts», «dentigerous cyst of inflammatory origin» y "follicular inflammatory-cyst» como key-words, full-text en inglés o español de los últimos 10 años. Resultados: Paciente 10 años de edad, presenta tumoración vestibular de zona de diente 5.3, de consistencia dura, asintomático y cubierto por mucosa normal. Diente asociado sin lesiones cariosas. La radiografía evidenció una extensa área radiolúcida bien definida, rodeando la corona del diente 1.3, desplazando y reteniéndolo. Tras evaluación radiográfica, los diagnósticos diferenciales fueron tumor-odontogénico-adenomatoide, queratoquiste y QD. La histopatología confirmó QFI. Originándose la lesión a partir de la pieza 5.4 con tratamiento pulpar. Conclusiones: Se debe considerar al QFI como una lesión similar a otros quistes inflamatorios (paradentales o radiculares). Se sugiere considerar en dentición mixta, que QFI y quiste radicular del diente temporal son la misma lesión y las diferencias en su aspecto clínico dependerán del tiempo de evolución y la relación de proximidad entre folículo y proceso inflamatorio del diente temporal.

PALABRAS CLAVE: Diente impactado, dentición mixta, quiste dentígero. 


\section{SUMMARY}

Objectives: To show the importance of histopathology in the diagnosis of follicular inflammatory cyst for a correct treatment and the relevance of a precise nomenclature. Material and Methods: Pubmed search, ScienceDirect, EBSCO-host "odontogenic cysts", "inflammatory dentigerous cyst" and "follicular inflammatory cyst" as keywords, full text in English or Spanish for the last 10 years. Results: A 10-year-old patient presented vestibular tumor of the tooth area 5.3, with a hard consistency, asymptomatic and covered by normal mucosa. Associated tooth without carious lesions. The radiograph evidenced a radiolucent area with well-defined limits, around the crown of the tooth 1.3, displacing and retaining it. After radiographic evaluation, the differential diagnoses were tumor-odontogenic-adenomatoid, keratocyst and QD. Histopathology confirmed QFI. Originating the lesion from piece 5.4 with pulp treatment. Conclusions: The QFI should be considered as an similar to other inflammatory (paradental or root) cysts. It is suggested to consider in mixed dentition, that QFI and radicular cyst of the temporal tooth are the same lesion and the differences in their clinical aspect will depend on the evolution time and the proximity relation between follicle and inflammatory process of temporal temporal.

\section{KEYWORDS: Odontogenic-cysts, dentigerous cyst of inflammatory origin, follicular inflammatory-cyst.}

\section{INTRODUCCIÓN}

Los quistes odontogénicos son de gran importancia dentro de las patologías orales y maxilofaciales, siendo a menudo las más frecuentes (1). Kramer definió un quiste como una cavidad patológica con un revestimiento epitelial, con un contenido líquido o acuoso, semi-líquido o gas, no estando relacionada con el acumulo de secreción purulenta (2). Muchos quistes orales y maxilofaciales son asintomáticos y suelen ser encontrados como hallazgos radiográficos.

En general, los quistes de la región oral y maxilofacial fueron clasificados en 1992 por Kramer, la que corresponde a la clasificación adoptada por la OMS. En ella se catalogan de acuerdo al origen de su componente epitelial y posteriormente a su patogénesis (3). Aquellos quistes que derivan su componente epitelial a partir de remanentes de tejidos dentarios se denominan "odontogénicos" los que a su vez se dividen, según su patogénesis, en inflamatorios y del desarrollo. Por otra parte, los quistes cuyo componente epitelial no es derivado de remanentes dentarios se clasifican como quistes del desarrollo "no odontogénicos”, también denominados fisurales $(4,5)$.

Dentro de la clasificación de quistes odontogénicos de la OMS, se incluyen en la categoría "origen inflamatorio" los quistes radiculares (lateral, residual y apical), el quiste de ubicación para-dental y el quiste vestibular-infectado de la mandíbula, sin embargo, no incluyen al quiste folicular inflamatorio (QFI), considerado la mayoría de las veces como un quiste dentígero (QD) catalogado como quiste del desarrollo (6).

El QFI se define como un quiste con desarrollo envolventede la porción coronaria de un diente permanente como resultado del compromiso intra-folicular de la inflamación periapical de un diente temporal suprayacente, con necrosis pulpar o tratamiento endodóntico parcial(7). Se presenta entre la primera y segunda década de vida, en edades comprendidas entre los 5 y 12 años, en la etapa de dentición -mixta. Su frecuencia es mayor en hombres, particularmente relacionada a molares deciduos de la zona mandibular izquierda, afectando a los premolares definitivos (8-10)

El objetivo del presente trabajo fue dar a conocer una revisión de la literatura acerca del QFI a propósito de un caso atípico donde la lesión no está en directa relación con la pieza temporal afectada y que al mismo tiempo presenta una ubicación extremadamente infrecuente (sector anterior del maxilar superior), lo que sugiere un reto diagnóstico y terapéutico. Por lo tanto, se pondrán en discusión características tanto imagenológicas como clínicas, los diagnósticos diferenciales y los diferentes opciones terapéuticas. Para la revisión de la literatura la búsqueda fue realizada en bases de datos electrónicas: PubMed, ScienceDirect, SciELO y Ebsco. Se utilizaron palabras claves como "Odontogenic Cysts, dentigerous inflammatory cyst, Folicullar inflammatory cyst, Deciduos teeth, 
primary dentition, mixed dentition, maxillary cyst, treatment y diagnosis”. Los filtros de la búsqueda fueron: publicaciones full text, en inglés o español, publicadas en los últimos 10 años.

\section{Reporte del caso}

Niño de 10 años de edad de género masculino, que al examen clínico evidenció un aumento de volumen vestibular en la región canina derecha de 3 centímetros de diámetro de consistencia firme, ubicado a 10 milímetros sobre el bermellón del labio superior y a 20 milímetros lateral al ala de la nariz.

$\mathrm{Al}$ examen intraoral se evidencia aumento de volumen vestibular, con límites bien definidos, asintomático, cubierto por mucosa sana, en relación al diente 5.3 clínicamente sin alteraciones, de evolución indeterminada. La evaluación radiográfica convencional evidenció una extensa área radiolúcida, unilocular de límites corticalizados, bien definida, asociada a la corona del diente 1.3, el cual se encontró desplazado y retenido. El diente 5.3 no evidenció signos de rizálisis, sin embargo el diente 5.4 evidenció un relleno opaco cameral, clínicamente presentaba una corona metálica preformada e historia de pulpotomía hace 5 años. Se solicitó un CBCT de la región, se obtuvo que la extensión de la lesión fue de $22 \mathrm{~mm}$ en sentido ocluso-apical, $17 \mathrm{~mm}$ en sentido antero-posterior y $22.3 \mathrm{~mm}$ de extensión mesio-distal con adelgazamiento de las corticales alveolares vestibular y lingual, en relación a las raíces de las piezas 1.2, 1.4, 5.3 y 5.4, encontrándose la pieza afectada desplazada y retenida en mesio-inclinación, próxima a la pared lateral de fosas nasales y piso de seno maxilar. El diente 5.4 evidenció extensa rizálisis, e inmediato a este se situaba la pieza 1.4, con erupción ectópica vestibularizada y en palatoversión (figura 1).

En primera instancia debido a la edad de presentación y la delimitación de la lesión más allá del límite amelocementario en ciertas zonas de la pieza 1.3, se sospechó más de un tumor odontogénico queratoquístico que de un quiste dentígero por lo que se procedió a realizar una punción y aspiración de la lesión para evaluar su contenido, gracias a la conducta cooperadora del paciente, la cirugía se realizó en el pabellón odontológico bajo anestesia local, donde se obtuvo una pequeña cantidad de líquido sanguinolento $(0,2 \mathrm{ml})$.
Al comprobar que la lesión no era sólida ni de contenido purulento o de queratina, se procedió a la apertura quirúrgica de la cavidad mediante un colgajo semilunar de espesor total. La tabla vestibular se encontraba adelgazada y sin signos de perforación. Se accedió a la lesión a través de una ventana ósea, se realizó la escisión completa del tejido que recubría la cavidad quística, posteriormente se efectuó un lavado profuso del lecho óseo con suero fisiológico estéril, concluyendo con el tratamiento quirúrgico con síntesis de sutura reabsorbible. El revestimiento quístico se fijó en formalina al $10 \%$ para su estudio histopatológico bajo la hipótesis diagnóstica de un QD (figura 2).

El examen histopatológico reveló características compatibles con un quiste de origen inflamatorio, evidenciándose un delgado epitelio ( 2 a 3 capas de espesor) de revestimiento escamoso estratificado no queratinizado. El tejido conectivo subyacente mostró un moderado infiltrado inflamatorio crónico compuesto principalmente por linfocitos y células plasmáticas, gran cantidad de fibras colágenas y vasos sanguíneos. No se mostraron evidencias de malignidad (figura 2). Confirmando como diagnóstico definitivo un QFI. Después de siete días, con la confirmación diagnóstica, se realizó la exodoncia de la pieza 5.4 con pulpotomía y se decidió conservar la pieza 5.3. Se evaluó la remisión de la lesión y la posible erupción espontánea del canino retenido mediante controles radiográficos periódicos cada 6 meses. En el primer control radiográfico se evidenció remodelado óseo de la lesión quística junto al notorio descenso de la pieza 1.3, luego a los 12 meses el canino afectado muestra una posición intraósea idéntica a la del canino contralateral, el tejido óseo muestra una cicatrización completa y no se ven signos de recidiva, mientras tanto el canino temporal mantuvo el espacio en la arcada durante este periodo. Actualmente está en evaluación ortodóntica para instalación de aparatos y tratamiento convencional (figura 3). 

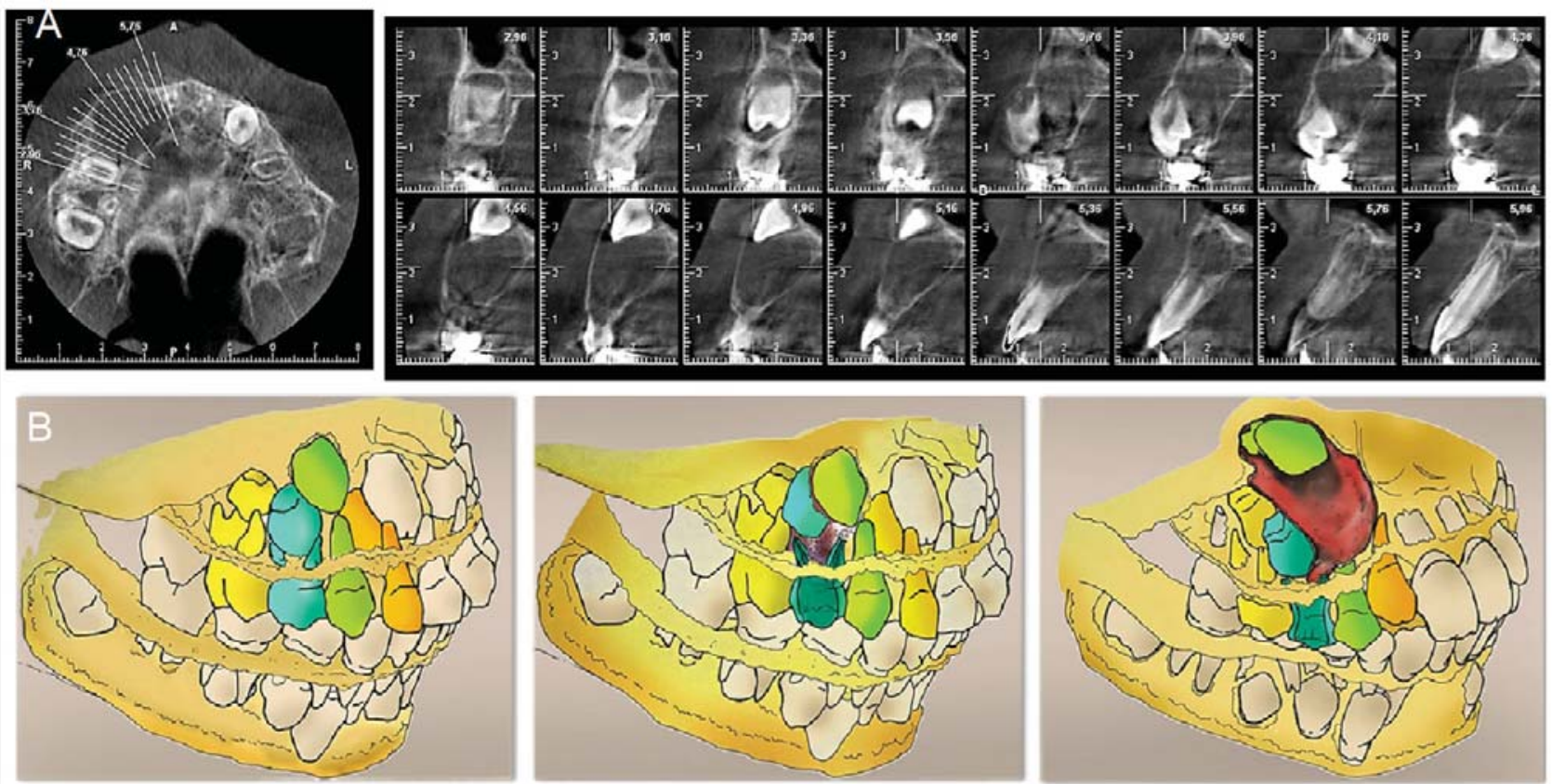

Figura 1. Análisis del caso : A) Cortes CBCT B) Representación gráfica de evolución del caso clínico desde un patrón extra-folicular
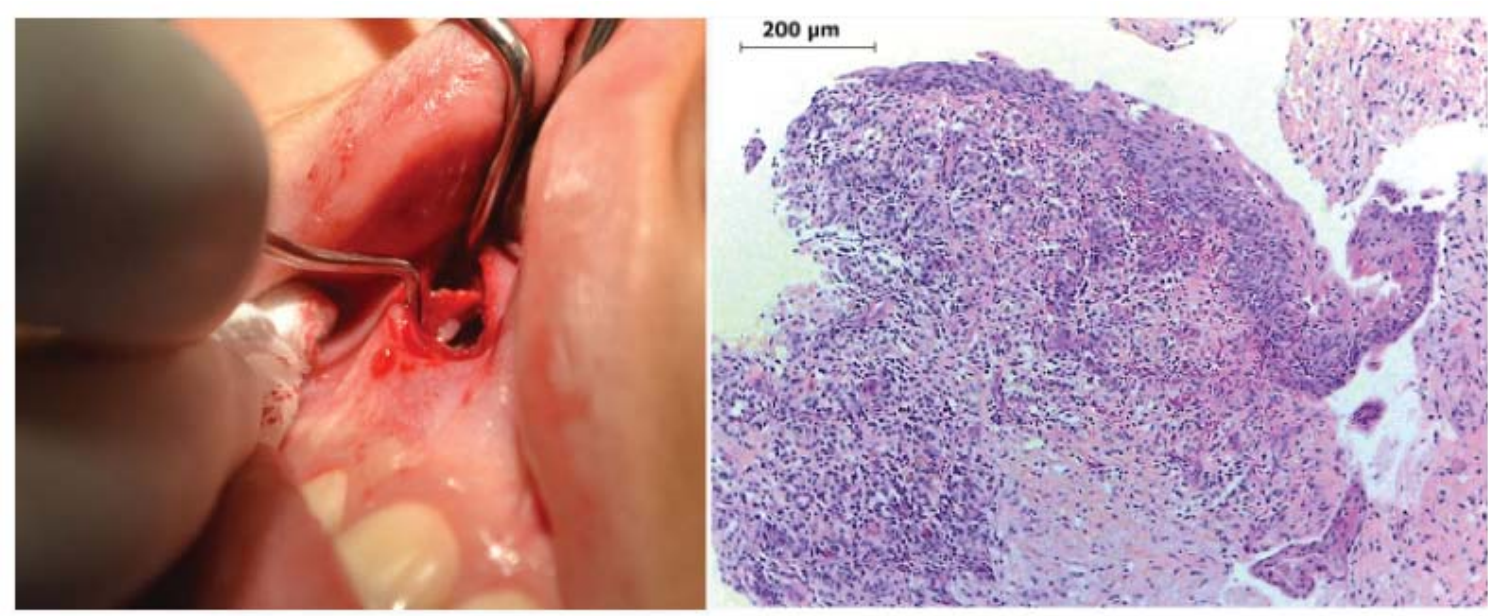

Figura 2. A) Enucleación B) Microfotografía Biopsia (HE) 10x 


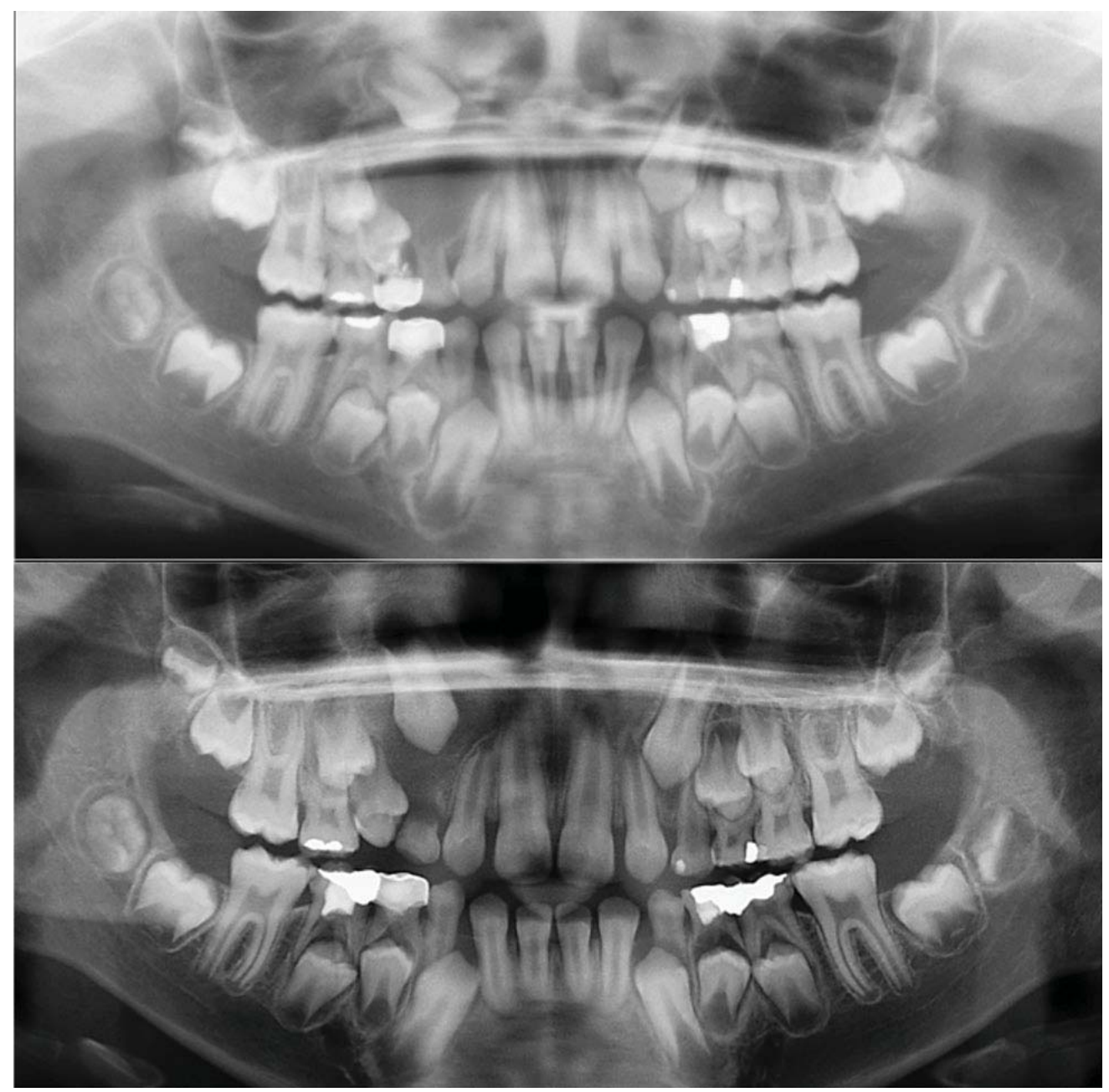

Figura 3. A) Radiografía Panorámica inicial B) Radiografía Panorámica control 6 meses

\section{DISCUSIÓN}

La formación de quistes en los maxilares requiere de tres elementos: una fuente de epitelio, un estímulo para la proliferación epitelial y la capacidad expansión quística con consecuente reabsorción ósea (5). El componente epitelial de los gérmenes dentarios se denomina órgano del esmalte y está involucrado en la formación del componente adamantino así como también en la iniciación de la formación de dentina. En la región del asa cervical del germen dentario es donde comienza el crecimiento apical de este, en lo que se conoce como la vaina epitelial de Hertwig, crucial para el desarrollo radicular. La lamina dental, el órgano del esmalte y la vaina epitelial de Hertwig degeneran a medida que se desarrolla el diente. Estos elementos se reducen a remanentes epiteliales cono- cidos como restos epiteliales de Malassez, el epitelio reducido del esmalte y las glándulas de Serres, los que están implicados en el origen de los quistes, mediados por mecanismos inflamatorios en el caso de los quistes de este origen. Por otra parte dan paso a quistes del desarrollo cuando son inducidos mediante mecanismos poco dilucidados, entre los cuales se consideran algunos procesos aberrantes en la formación dentaria e inclusive anomalías genéticas (3).

La frecuencia de quistes odontogénicos en la población pediátrica es relativamente baja (11). Tanto los quistes del desarrollo como los inflamatorios manifiestan un crecimiento lento y con tendencia a la expansión ósea. Sin embargo, a pesar de su comportamiento benigno, estas lesiones pueden alcanzar grandes tamaños si no son diagnosticadas y tratadas 
oportunamente (12). Además, el correcto diagnóstico es esencial para proponer un tratamiento quirúrgico ideal y un seguimiento adecuado (13). Padmakumar et al., en su estudio retrospectivo de 12 años reportaron que la incidencia de los quistes maxilares en pacientes pediátricos fue predominante en quistes del desarrollo con un $63,2 \%$ mientras que los de origen inflamatorio se remitieron a un 33.6\% (14). Hallazgos similares a los reportados por Bodner et al., (15) en el 2012 donde los quistes del desarrollo alcanzaron un $44 \%$ mientras los de origen inflamatorio se consignaron en un $17 \%$ y el $66.7 \%$ de incidencia de quistes del desarrollo en niños y adolescentes reportado por Da Cruz et al., (16) un año antes en su estudio retrospectivo de 21 años.

El quiste QD también conocido como quiste folicular, es el quiste odontogénico del desarrollo más común, corresponden a un $18 \%$ de todos los quistes odontogénicos y un 58\% de los del desarrollo (17). Definido por la OMS como un quiste odontogénico generadopor el acúmulo de fluido entre la capa epitelial reducida y la porción coronaria de un diente no erupcionado e impactado (18), no ocasionado por estímulos inflamatorios (19) adherido al límite amelocementario, delimitado en este punto por los límites del órgano del esmalte (3). Una de las características clínicas más comunes de un QD es la expansión de la cortical vestibular(20). La presión hidrostática causada por la acumulación de fluido resulta en expansión del folículo dental y la reabsorción ósea $(3,21)$.

Durante años, las lesiones osteolíticas formadas alrededor de la corona de premolares no erupcionados en dentición mixta se consideraron como QD(22-24). Los autores defienden que estos quistes se desarrollan desde un patrón extra-folicular en cual el estímulo de un diente necrótico temporal supra adyacente causa la separación del epitelio reducido del esmalte de la superficie del esmalte resultando en la formación de un quiste (25). Estas declaraciones agregan un origen inflamatorio a los QD que se consideran como quistes del desarrollo. Autores sugieren que el QD puede surgir como resultado de una inflamación de cualquier fuente, pero usualmente de dientes deciduos no vitales, con afección del diente permanente sucesor. Ellos proponen la existencia de dos tipos de QD: uno del desarrollo y otro de naturaleza inflamatoria. Los primeros son originados en dientes definitivos, usualmente ocasionados por impactación dentaria. Por otra parte, los inflamatorios son de naturaleza reaccional, originados en dientes en evolución intraósea inmaduros como resultado de inflamación proveniente de un diente deciduo no vital u otro medio de difusión de procesos inflamatorios que involucran el folículo del diente definitivo(26-28). Benn y Altini describen tres mecanismos posibles para la histogénesis de un QD inflamatorio (27): El primer mecanismo debido a la inflamación secundaria de un QD del desarrollo de origen intra-folicular, siendo usualmente la fuente de inflamación un diente no vital. El segundo mecanismo es a partir de un quiste radicular, formado en los ápices del diente temporal desvitalizado y el sucesor definitivo erupciona dentro del quiste apical generando un quiste dentígero de origen extra-folicular. El tercer tipo es a partir de una inflamación periapical proveniente de un diente temporal no vital u otra fuente, la que se esparce y envuelve el folículo permanente influenciando un QD.

En la mayoría de estudios y series de casos se categorizan indistintamente los QFI y los QD, resultando en rangos etarios bastante amplios y en ocasiones altos porcentajes de incidencia en pacientes pediátricos llegando a un 30\% del total según algunos autores ${ }^{(1)}$. Se describe que la mayor incidencia de QD ocurre entre la $2^{\mathrm{a}}$ y $3^{\mathrm{a}}$ década de vida, incluso entre la $3^{\mathrm{a}} \mathrm{y}$ $4^{a}$ década (18). Con una incidencia ligeramente mayor en hombres de 1.6:1. Está principalmente asociado a terceros molares inferiores y caninos superiores incluidos (13) raramente afectando otros dientes. La frecuencia de QD en estos sitios puede ser explicada por el hecho de que los terceros molares mandibulares y los caninos maxilares son los dientes con una frecuencia de impactación mayor(13). A diferencia del QFI que se presenta entre la primera y segunda década de vida, en edades comprendidas entre los 5 y 12 años, en la etapa de dentición-mixta, que afecta a molares deciduos de la zona mandibular izquierda, comprometiendo los premolares definitivos(8-10). La asociación del QFI con los segundos molares deciduos mandibulares se explica mediante dos teorías, la primera postula que este molar es el que tiene mayor suceptibilidad a caries, mientras la segunda explica la ocurrencia por una relación de mayor proximidad entre el germen del premolar permanente con este molar temporal (29).

Clínicamente se manifiesta de forma variable según el tamaño de la lesión y su tiempo de evolución, normalmente se evidencia como un aumento de volumen 
de consistencia dura cubierto por mucosa indemne, causado por el abombamiento de la tabla alveolar vestibular (9). Movilidad dentaria, desplazamiento de dientes y sensibilidad pueden estar presentes si el quiste alcanza un tamaño superior a $2 \mathrm{~cm}$ de diámetro (30). El diagnóstico diferencial de un folículo ensanchado debido a infección se basa en el hecho de que la radiolucidéz peri coronaria sea $>2,5 \mathrm{~mm}$ en su diámetro mayor(31) y se establece que si el espacio folicular es $>4 \mathrm{~mm}$ corresponde interpretarlo directamente como un quiste (1).

Por lo general este tipo de lesión es asintomática y el $22 \%$ es detectado en evaluaciones rutinarias según Lustig et al., (29), siendo el resto evidenciadas cuando presentan sintomatología aguda dolorosa de origen infeccioso, con aumento local de temperatura, secreción purulenta y aumento de volumen facial. Autores postulan un tiempo de 2 a 5 años para el desarrollo del quiste desde la realización de la pulpotomía, dependiendo de factores como el tipo de hueso, la edad del paciente y localización del diente (32). La frecuencia de QD en niños se ha reportado baja en la literatura, Shears estimó que es de un 9\% (33), mientras Donath describió que cerca del 4\% de los QD ocurren dentro de la primera década de vida (34). Sin embargo esto se contrapone con algunos resultados en la literatura que relatan que en niños de 2 a 14 años los QD representan un 49\% de las lesiones quísticas intraóseas(35). Por ejemplo, Shibata et al., muestran que la edad de hallazgo de quistes dentígeros fue generalmente entre los 9-11 años (24). Existe numerosa literatura de quistes dentígeros reportados en pacientes menores a 15 años, además en pacientes pediátricos con dentición mixta. Estudios donde los QFI son considerados indistintamente como QD adjudicándoles un origen inflamatorio, lo que sería incorrecto desde el punto de vista etiopatogénico.

\section{a) Etiopatogenia del QFI y correcta clasificación.}

Main en 1985 definió el término de "quiste folicular inflamatorio" bajo la hipótesis de que el quiste se produce en relación a la porción coronal del diente permanente como resultado del esparcimiento intra folicular de la inflamación periapical de un diente temporal supra yacente (7). Sin embargo, esto pudo generar un problema semántico para la clasificación ya que en la literatura internacional el QD también es conocido como quiste folicular, resultando el término QFI un sinónimo de QD inflamatorio (mayormente utilizado en la literatura), lo que dificulta obtener bi- bliografía precisa para una u otra lesión. Estas lesiones aunque clínicamente son muy similares, poseen un origen y patogénesis distinta, por lo tanto proponemos que debería restringirse la denominación de quiste folicular a este tipo de lesiones inflamatorias, limitando el término dentígero exclusivamente al quiste del desarrollo. En el 2012 Martínez et al., propusieron una nueva clasificación para los quistes inflamatorios de los maxilares, agregando el QFI a los quistes de origen inflamatorio, antes catalogado como una variante del QD del desarrollo. Los autores propusieron clasificar de forma separada a los quistes asociados a inflamación periapical generada por necrosis pulpar de los dientes deciduos asociadosa inflamación durante el proceso de erupción, en donde estaría el QFI(36). La evidencia clínica, radiográfica y microscópica de la lesión osteolítica descrita permite su categorización como una lesión quística inflamatoria, con características de un quiste inflamatorio y no del desarrollo, originada por la inflamación del diente temporal no vital (37).

Mediante un análisis preciso de estudios nuevos y antiguos acerca de este tema, no se puede establecer que indudablemente todas las lesiones radiolúcidas descritas por Bloch-Jorgensen(38), Azaz y Shteyer(22), Shaw et al., (23), Shibata et al., (24) y Benn y Altini(27), pueden ser catalogadas como quistes dentígeros. Algunos de ellos reportan varios casos en donde no existe un criterio fuerte de diagnóstico diferencial con un quiste radicular y un QD. Shaw et al., (23) postularon que "si un quiste primario peri radicular se estaba presente en el proceso de desarrollo de su diente sucesor es probable que se lleve a cabo la unión del folículo con el quiste, resultando en la continuidad del epitelio reducido del esmalte y el revestimiento epitelial del quiste. Respecto a esto los autores consideraron la posibilidad de que el "quiste dentígero" descrito en la dentición mixta fuera desarrollado a partir de la progresión de un quiste radicular preexistente (37). Compartiendo más características histológicas con un quiste radicular que con un quiste dentígero.

Benn y Altini mostraron una serie de 15 pacientes con patología oral clasificable como QD de origen inflamatorio, basados en hallazgos clínicos, radiográficos y microscópicos. Los microscópicos mostraron numerosas zonas de la pared del quiste revestidas por una capa de 2 a 3 células de grosor, cuboidales, similares al epitelio reducido del esmalte, sin embargo el epitelio se continuaba con uno hiperplásico escamoso estratificado no queratinizado de grosor variable, al- 
gunas veces con papilas anastomóticas (27). Muchos de estos casos estaban revestidos completamente con este epitelio hiperplásico con abundante infiltrado inflamatorio, siendo imposibles de diferenciar con quistes radiculares, paradentales y otros quistes inflamatorios. Los autores defendieron la hipótesis de que la formación del QD ocurrió como resultado al exudado inflamatorio crónico de dientes primarios no vitales o con tratamientos pulpares, que causó la separación del epitelio reducido del esmalte del diente no erupcionado, inclusive considerando la posibilidad de que los quistes radiculares desarrollados en los ápices de dientes deciduos no vitales se expanden llegando a fusionarse con el folículo de los dientes sucesores no erupcionados (37).

En cabeza y cuello los quistes más típicos son los radiculares, se desarrollan cuando la inflamación pulpar difunde hacia el ápice o zona radicular lateral y causan la proliferación de los restos de Malassez, restos celulares que dan pie a la formación del ligamento periodontal, dando origen a una cavidad quística con revestimiento epitelial(18). Aunque los mecanismos de desarrollo de los quistes radiculares son idénticos en la dentición temporal y permanente, los quistes primarios originados en dientes temporales son considerados extremadamente raros. Lustmann and Shear reportaron una incidencia de solo un $0,5 \%$ en su estudio de 25 años (39). La rara ocurrencia de quistes radiculares en dientes primarios fue rechazada por Mass et al., (40) en su estudio de 49 lesiones radiolúcidas en molares primarios donde un $73.5 \%$ fue diagnosticado como quistes radiculares y un $26.5 \%$ como granulomas. Los autores insisten en la idea que los quistes radiculares asociados a dientes deciduos no son raros, sino que están escasamente reportados en la literatura debido a que la mayoría de estas lesiones se subestiman clínicamente y la mayoría se resuelve luego de la exodoncia del diente temporal afectado, sin proceder a un análisis histopatológico. Muchos factores pueden estar implicados en la formación de quistes radiculares en dientes primarios: alta prevalencia de caries, estímulos de terapias pulpares, la existencia de canales accesorios, gran número de remanentes epiteliales en la dentición mixta y finalmente la alta actividad proliferativa de la gente joven con dentición mixta. $(18,41)$.

La correcta diferenciación entre un quiste inflamatorio (radicular) y un quiste dentígero en la dentición mixta es imposible desde la clínica, imagenología e histopatología. Basándose en lo descrito por De Cas- tro y Campos (37) y por Lustmann y Shea r(39), se debe considerar que un quiste radicular y un eventual "quiste dentígero" en dentición mixta son de hecho la misma lesión en distintas etapas de desarrollo, denominado correctamente quiste folicular inflamatorio.

Respecto al caso presentado, por sus características representa un reto diagnóstico. En un comienzo no se consideró un quiste de origen inflamatorio como el QFI, ya que el canino temporal se encontraba en buen estado y sin alteraciones, descartando la posibilidad de que el desarrollo del quiste asociado al canino permanente fuera consecuencia de la necrosis pulpar de su predecesor temporal, además de su escasa presentación en sector anterior del maxilar superior. Por otro lado el diagnóstico histopatológico determinó la naturaleza inflamatoria de la lesión y la conducta a seguir. La hipótesis respecto de por qué no se vio involucrado el premolar definitivo siendo la pieza más cercana al molar temporal con pulpotomía, fue que existe la posibilidad de que efectivamente se haya visto involucrado en un inicio en el QFI, pero es probable que por su posición próxima a erupcionar haya generado una comunicación de la corona con el medio oral, por otro lado, también existe la posibilidad de que su saco folicular estuviera lo suficientemente fibroso para no afectarse en mayor medida, por lo que en definitiva el diente afectado fue el canino superior adyacente, tomando en consideración que estas lesiones de tipo inflamatorio pueden desarrollarse a partir de un patrón extra-folicular de inflamación, posiblemente extendiéndose desde el premolar próximo a su erupción o directamente desde la lesión apical del diente temporal, generando una continuidad entre el saco folicular del canino y el quiste, resultando finalmente en la movilización e impactación de este. Además se podría asociar que los QFI debido a su naturaleza inflamatoria, no se limitan estrictamente al límite amelocementario como un QD, sino que pueden extenderse más allá, dependiendo de la magnitud del proceso inflamatorio, la extensión y la evolución de la lesión, característica que se corresponde con otros quistes inflamatorios como los quistes paradentales.

\section{b) Opciones terapéuticas}

El tratamiento de los quistes inflamatorios, incluyendo los quistes radiculares, es remover la causa de la inflamación, ya sea pulpa necrótica o un diente con terapia pulpar y en ciertos casos enucleación del revestimiento quístico también puede ser requerida ${ }^{(4)}$. La necesidad de un adecuado diagnóstico de la lesión 
quística y características clínicas locales del paciente, adquieren un rol fundamental para la correcta elección del tratamiento. La literatura muestra que la enucleación del QFI asociado a la extracción del diente impactado, es el principal tratamiento para prevenir recurrencia, sin embargo, es considerada como una modalidad de tratamiento agresiva, por la simple razón de que si existe capacidad eruptiva inherente al desarrollo radicular del diente afectado, el manejo conservador debiera ser la alternativa de preferencia. Los objetivos de un tratamiento conservador son disminuir la morbilidad del paciente, siendo la descompresión y marsupialización los tratamientos de elección sobre las resecciones quirúrgicas y enucleaciones que incluyen gérmenes dentarios (4). La marsupialización es un tratamiento donde se realiza una apertura de la pared quística para posteriormente vaciarlo, estableciendo una conexión con la cavidad oral, seno maxilar o cavidad nasal. Esta opción es de elección cuando existe espacio para la erupción de los dientes impactados. Se ha reportado que la tasa de erupción luego de la marsupialización varía en rangos de un 31 a un 89,4\% (42). Las radiografías panorámicas se utilizan para predecir la erupción del diente luego de la marsupialización en algunos estudios, utilizando como parámetros la edad y el género del paciente, profundidad cuspídea, eje de angulación del diente, el espacio interdental y tamaño de la impactación. Resultados de estudios demuestran que la edad y la profundidad de un diente asociado a un QFI son factores importantes para la erupción(43). Mientras otros se enfocan en la relación que existe entre la capacidad de erupción de un diente impactado asociado con un QFI según su nivel de formación radicular(44). Chi Yang et al., en el 2013 evaluaron los factores involucrados en la predicción de la capacidad de erupción espontánea luego de la marsupialización de QFI. En sus resultados obtuvieron que un 8.1 meses en promedio fueron requeridos para que los dientes impactados erupcionaran espontáneamente en el grupo asociado a quistes. Por otro lado demostró que los dientes asociados a QFI requieren un menor tiempo para erupcionar que en el grupo control, sin lesiones quísticas. Resultados que se condicen con los de Miyawaki et al., (45), que proponen que la velocidad de erupción es consistente con la rápida formación ósea, la que es acompañada por una evidente disminución de la presión intraquística, promoviendo una rápida erupción del diente impactado. También encontraron que la angulación de los dientes fue significativamente ma- yor en el grupo con QFI, sin afectar su capacidad de erupción. Por lo tanto propusieron, según los resultados obtenidos, que el género, edad, profundidad cuspidea, la angulación del diente y espacio de erupción no fueron factores de riesgo significantes en la erupción de premolares impactados afectados por QFI en pacientes preadolescentes. Sin embargo, el nivel de formación radicular podría jugar un rol importante en la erupción de estos dientes, por lo que se recomienda que la marsupialización/descompresión debe ser efectuada antes de la formación radicular completa, siendo este tratamiento efectivo en preservar, permitir y promover la erupción en dientes impactados asociados a QFI (42).

En el caso reportado en esta investigación, se optó por realizar la descompresión y escisión del epitelio quístico, con posterior exodoncia de la pieza temporal afectada, sin realizar una marsupialización que comunique la extensa cavidad patológica con la cavidad oral, al evaluar la edad del paciente y tamaño del quiste, además de la profundidad del canino en el macizo facial. Esto permitió en base a lo descrito anteriormente, disminuir la presión intraquística y favorecer en gran medida el proceso de erupción del canino impactado y al mismo tiempo habiendo eliminando completamente el epitelio quístico, con resultados excelentes, un post operatorio sin sintomatología y sin compromiso estético ni emocional del paciente.

\section{CONCLUSIONES}

La extracción del diente deciduo, la enucleación de la membrana quística, y mantener en observación el proceso de erupción del diente impactado, definen la línea de tratamiento de elección para los QFI de gran extensión, considerando estos factores, no es recomendable la exodoncia del diente incluido afectado, a menos de que el caso y la ubicación del germen lo ameriten. Es de suma importancia tener en cuenta cuan comprometidas están las estructuras anatómicas y la posición del diente permanente al evaluar la forma de ser reubicado, pudiendo ser mediante manejo ortodóntico-quirúrgico según el caso. Imágenes radiográficas de las lesiones radio lúcidas de los huesos maxilares pueden compartir muchas características, lo que puede dificultar el diagnóstico y la elección de tratamientos idóneos. Por lo tanto la confirmación diagnóstica mediante la histopatología es fundamental para poder definir un plan de tratamiento. 
Respecto a la nomenclatura de esta lesión se debería emplear exclusivamente el término "folicular" para quiste de origen inflamatorio (QFI) y el término "dentígero" exclusivamente para el quiste del desarrollo (QD), evitando así la denominación del QFI como quiste dentígero inflamatorio. Además se debe considerar al QFI como una lesión indistinta a otros quistes inflamatorios como quistes paradentales o quistes radiculares. Por lo tanto, se sugiere considerar en la dentición mixta, que un QFI y un quiste radicular de la pieza temporal asociada, corresponden a la misma lesión y que las diferencias en su aspecto clínico dependen exclusivamente del tiempo de evolución de la lesión y de la relación de proximidad entre el folículo y el proceso inflamatorio proveniente del diente temporal.

\section{Correspondencia:}

\section{Dr. Emilio Moreno Apablaza}

Correo electrónico: Emilio.moreno.a@gmail.com

\section{REFERENCIAS BIBLIOGRÁFICAS}

1. Jones AV, Franklin CD.An analysis of oral and maxillofacial pathology found in adults over a 30-year period. J Oral Pathol Med. 2006; 35(7):392-401. doi: http:// doi.wiley.com/10.1111/j.1600-0714.2006.00451.x

2. Kramer I. Changing views on oral disease. Proc R Soc Med. 1974;67(4):271-6.

3. Shear M, Speight P. Cysts of the oral and maxillofacial regions. Oxford: Blackwell Munksgaard; 2007.

4. Martin L, Speight PM. Odontogenic cysts. Diagnostic Histopathology. 2015;21(9):359-369. doi: http:// dx.doi.org/10.1016/j.mpdhp.2015.07.005

5. Lima EDNDA, Maia CADM, Gurgel AC, de Oliveira PT, de Medeiros AMC. Conservative management of dentigerous cyst in a child. Int J Pediatr Otorhinolaryngol Extra. 2013;8(1):e1-4. (Citado el 3 de octubre del 2017) Disponible en: http://linkinghub.elsevier. com/retrieve/pii/S1871404812000524

6. EI-Naggar AK, Chan JK.C., Grandis J.R., Takata T. SPJ. WHO Classification of Head and Neck Tumours. 4th ed. IARC: Lyon 2017.p. 347.

7. Main DM. Epithelial jaw cysts: 10 years of the WHO classification. J Oral Pathol. 1985;14(1):1-7.

8. Motamedi MHK, Talesh KT. Management of extensive dentigerous cysts. Br Dent J. 2005;198(4):203-6.

9. Neville B, Damm D. Oral and Maxillofacial Pathology. Louis, Mo,USA: Saunders; 2016.

10. Kozelj V, Sotosek B. Inflammatory dentigerous cysts of children treated by tooth extraction and decompression--report of four cases. Br Dent $\mathrm{J}$. 1999;187(11):587-90.
11. Deboni M, Brozoski M, Traina A,Acay R, Naclério-Homem M. Surgical management of dentigerous cyst and keratocystic odontogenic tumor in children: a conservative approach and 7-year follow-up. J Appl Oral Sci. 2012; 20(2): 282-285. doi: 10.1590/ S1678-77572012000200025

12. Ishihara Y, Kamioka H, Takano-Yamamoto T, Yamashiro T. Patient with nonsyndromic bilateral and multiple impacted teeth and dentigerous cysts. Am J Orthod Dentofacial Orthop. 2012;141(2):228-41. doi: 10.1016/j.ajodo.2010.02.043

13. Selvamani M, Donoghue M, Basandi PS. Analysis of 153 cases of odontogenic cysts in a South Indian sample population: a retrospective study over a decade. Braz Oral Res. 2012; 26(4):330-4.

14. Padmakumar SK. Cysts of the Jaws in Pediatric Population : A 12-Year Institutional Study. Oral \& Maxillofacial Pathology Journal. 2014; 5(2) :532-7.

15. Manor E, Kachko L, Puterman MB, Szabo G, Bodner L. Cystic lesions of the jaws - a clinicopathological study of 322 cases and review of the literature. Int J Med Sci. 2012;9(1):20-6.

16. Serra VG, Conde DM, Vera R, et al. Odontogenic cysts in children and adolescents : a 21-year retrospective study. Brazilian J Oral Sci. 2012;11(2):81-3.

17. Jones A V, Craig GT, Franklin CD. Range and demographics of odontogenic cysts diagnosed in a UK population over a 30-year period. J Oral Pathol Med. 2006;35(8):500-7.

18. Kramer IRH, Pindborg JJ, Shear M. Histological Typing of Odontogenic Tumours. Berlin, Heidelberg: Springer Berlin Heidelberg; 1992 (Citado el 3 de octubre del 2017) Disponible en: http://link.springer. com/10.1007/978-3-662-02858-2

19. Tamgadge A, Tamgadge S, Bhatt D, Bhalerao S, Pereira T, Padhye $\mathrm{M}$. Bilateral dentigerous cyst in a non -syndromic patient: Report of an unusual case with review of the literature. J Oral Maxillofac Pathol. 2011;15(1):91-5. doi: 10.4103/0973-029X.80017

20. Hu Y-H, Chang Y-L, Tsai A. Conservative treatment of dentigerous cyst associated with primary teeth. Oral Surg Oral Med Oral Pathol Oral Radiol Endod. 2011; 112(6):e5-7. doi: 10.1016/j.tripleo.2011.03.041

21. Tilakraj TN, Kiran NK, Mukunda KS, Rao S. Non syndromic unilateral dentigerous cyst in a 4-year -old child: A rare case report. Contemp Clin Dent. 2011;2(4):398-401. doi: 10.4103/0976-237X.91813

22. Azaz B, Shteyer A. Dentigerous cysts associated with second mandibular bicuspids in children: report of five cases. ASDC J Dent Child. 1973;40(1):29-31.

23. Shaw W, Smith M, Hill F. Inflammatory follicular cysts. ASDC J Dent Child. 1980;47(2):97-101. 24.Shibata Y, Asaumi J, Yanagi Y, et al. Radiographic examination of dentigerous cysts in the transitional dentition. Dentomaxillofac Radiol. 2004;33(1):17-20. 
24. Bozdogan E, Cankaya B, Gencay K, Aktoren O. Conservative management of a large dentigerous cyst in a 6-year-old girl: a case report. J Dent Child (Chic). 201; 78(3):163-7.

25. Narang RS, Manchanda AS, Arora P, Randhawa K. Dentigerous cyst of inflammatory origin-a diagnostic dilemma. Ann Diagn Pathol. 2012;16(2):119-23. doi: 10.1016/j.anndiagpath.2011.07.004.

26. Benn A, Altini M. Dentigerous cysts of inflammatory origin. A clinicopathologic study. Oral Surg Oral Med Oral Pathol Oral Radiol Endod. 1996;81(2):203-9.

27. Prabhu NT, Rebecca J, Munshi AK. Dentigerous cyst with inflammatory etiology from a deciduous predecessor--report of a case. J Indian Soc Pedod Prev Dent. 1996;14(2):49-51.

28. Lustig JP, Schwartz-Arad D, Shapira A. Odontogenic cysts related to pulpotomized deciduous molars: clinical features and treatment outcome. Oral Surg Oral Med Oral Pathol Oral Radiol Endod. 1999; 87(4):499503.

29. Bodner L, Woldenberg Y, Bar-Ziv J. Radiographic features of large cystic lesions of the jaws in children. Pediatr Radiol. 2003; 33(1):3-6.

30. Adelsperger J, Campbell JH, Coates DB, Summerlin DJ, Tomich CE. Early soft tissue pathosis associated with impacted third molars without pericoronal radiolucency. Oral Surg Oral Med Oral Pathol Oral Radiol Endod. 2000;89(4): 402-6.

31. Aguiló L, Gandía JL. Dentigerous cyst of mandibular second premolar in a five-year-old girl, related to a non-vital primary molar removed one year earlier: a case report. J Clin Pediatr Dent. 1998; 22(2):155-8.

32. Shear M. Cysts of the oral regions. 3rd edition. Oxford: Oxford University Press; 1992. P.75-89.

33. Donath K. Odontogenic and nonodontogenic jaw cysts. Dtsch Zahnarztl Z. 1985;40(6):502-9.

34. Regezi A, Sciubba J, Jordan R. Oral Pathology, Clinical Pathologic Correlations. 6th edition. St. Louis, Mo,USA: Saunders; 2012.

35. Klingbeil DEF, Bucal P, Meira GDEF. Classification of inflammatory cysts of the jaws, new proposal. Oral Surg Oral Med Oral Pathol Oral Radiol. 2012; 114(4):e56. (Citado el 3 de octubre del 2017) Disponible en: http://linkinghub.elsevier.com/retrieve/pii/ S2212440312010875

36. De Castro LA, Campos Maia SR. Maxillary osteolytic lesion in a 10-year-old girl: A dentigerous or radicular cyst? A case report and discussion. Rev Port Estomatol Med Dent e Cir Maxilofac. 2012; 53(1):24-8. doi: http://dx.doi.org/10.1016/j.rpemd.2011.10.004

37. Bloch-Jorgensen K. Follicular cysts. Dent Cosmos. 1928; 70(708):11.

38. Lustmann J, Shear M. Radicular cysts arising from deciduous teeth. Review of the literature and report of 23 cases. Int J Oral Surg. 1985;14(2):153-61.
39. Mass E, Kaplan I, Hirshberg A. A clinical and histopathological study of radicular cysts associated with primary molars. J Oral Pathol Med. 1995;24(10):45861.

40. Grundy GE, Adkins KF, Savage NW. Cysts associated with deciduous molars following pulp therapy. Aust Dent J. 1984;29(4):249-56.

41. Qian WT, Ma ZG, Xie QY, Cai XY, Zhang Y, Yang C. Marsupialization facilitates eruption of dentigerous cyst-associated mandibular premolars in preadolescent patients. J Oral Maxillofac Surg. 2013;71(11):182532. doi: http://dx.doi.org/10.1016/j.joms.2013.06.223

42. Fujii R, Kawakami M, Hyomoto M, et al. Panoramic findings for predicting eruption of mandibular premolars associated with dentigerous cyst after marsupialization. J Oral Maxillofac Surg. 2008; 66(2): 272-6. (Citado el 3 de octubre del 2017) Disponible en: http://linkinghub.elsevier.com/retrieve/pii/ S0278239107014930

43. Marks SC, Schroeder HE. Tooth eruption: Theories and facts. Anat Rec. 1996; 245(2):374-93.

44. Miyawaki S, Hyomoto M, Tsubouchi J, Kirita T, Sugimura M, Lucas R, et al. Eruption speed and rate of angulation change of a cyst-associated mandibular second premolar after marsupialization of a dentigerous cyst. Am J Orthod Dentofac Orthop. 1999; 116(5):578-84. (Citado el 3 de octubre del 2017) Disponible en: http://linkinghub.elsevier.com/retrieve/pii/S0889540699701927

Recibido: 07-06-2017 Aceptado: 29-09-2017 